\title{
La réception de la propagande d'Auguste chez Sénèque le Rhéteur
}

\author{
MARCOS TORRI \\ Doutorando pelo Institut d'Archéologie \\ et des Sciences de l'Antiquité - Université de Lausanne
}

RESUMO: A ambigüidade e os paralelismos históricos presentes nos discursos de Sêneca o Velho sobre Augusto revelam que a lembrança do Princeps não se enquadra nos grandes temas da propaganda imperial. Através destes recursos o autor exprime uma espécie de oposição política.

PALAVRAS-CHAVE: Roma; Principado; Augusto; Sêneca o Velho; memória; propaganda; oposição política.

La propagande d'Auguste est divisée en deux parties: la première, que nous pouvons situer entre 44 et 36 av.J.-C., comprend la féroce recherche du pouvoir et la vengeance contre les assassins de Jules César; elle est caractérisée par la divinité choisie par Auguste: Mars Ultoris. La deuxième commence avec le choix d'Apollon en 36 av. J.-C. (Suet. Div. Aug. 29; Properc. Eleg. 4,6,11-68), passe par la guerre contre Marc Antoine et, après la victoire d'Actium en 31 av. J.-C., comprend tout un projet d'image travaillée pour la postérité dans la littérature, architecture et politique, jusqu'à la mort du Princeps en 14 ap. J.-C. Ce large programme a été conçư pour préserver le régime et l'image de son fondateur; il peint Auguste comme le pacificateur et le pieux restaurateur de la République, comme l'aboutissement naturel et nécessaire de l'histoire de Rome, tel que nous pouvons le voir dans la suite de personnages historiques sur les portiques du forum d'Auguste ou dans les reliefs de l'Ara Pacis, mais le meilleur propagandiste, celui auprès duquel le lecteur contemporain peut trouver le moins d'ambiguïtés possibles est Auguste lui-même'. Je propose de regarder les sources littéraires à la lumière de cette propagande. Est-ce que les auteurs en ont emprunté des éléments? Ont-ils été indifférents, opposants ou de simples passeurs d'idées reçues? Comment cette image voulue est-elle présente dans des esprits indépendants comme celui de Sénèque le Rhéteur?

1 L'index rerum a se gestarum ou Res Gestae. Voir notamment Malitz, J: Autobiographie und Biographie römischer Kaiser im 1. Jh. N.Chr. In: Weber, G. (Org)/ Propaganda Selbstdarstellung - Repräsentation in römischen Kaiserreich des 1. Jhs. N.Chr. Stuttgart: 
L'histoire et la réception de cette propagande d'Auguste, c'est-à-dire du portrait qu'il a préparé pour la postérité durant plus de 40 ans de pouvoir, sont souvent décrites à partir de préjugés concernant l'appartenance sociale des sources historiques étudiées. Il est, en fait, difficile d'écarter cette conviction que chaque groupe social dans la Rome ancienne avait son avis précis sur Auguste. Ces idées reçues et ces réponses déjà prêtes de l'historiographie nuisent à l'intérêt de l'histoire vivante, interdisent la juste appréciation de ses acteurs et empêchent le progrès de la réflexion.

Je présenterai d'abord l'Oratorum et rhetorum sententiae, divisiones, colores (“Avis, plans, couleurs des orateurs et des rhéteurs") de Sénèque le Rhéteur en y relevant les mentions d'Auguste. Chaque mention sera suivie d'une réflexion sur la place de ce personnage et de son régime dans l'œuvre. A partir de ces réflexions, nous examinerons d'autres questions soulevées par Sénèque qui, à mon avis, se rapportent aussi à Auguste et au Principat.

Sénèque a commencé à écrire son ouvrage en 37 ap. J.-C.; il répondait à la demande de ses enfants qui voulaient savoir ses sentiments sur les déclamateurs dont il avait été le contemporain et l'interrogeaient sur ces hommes dont l'existence ne s'était pas prolongée jusqu'à leur époque. Derrière ses enfants, comme nous le verrons, il songeait naturellement au public, dont il avait éprouvé l'admiration². Mises à part ces considérations, nous ne répéterons pas ici d'anciennes idées reçues et nous ne dirons pas que l'ouvrage avait la fonction de préparer les gens à l'éloquence judiciaire: les causes développées étaient trop fictives et insolites pour le barreau. La préoccupation de l'auteur était d'éduquer ses auditeurs en histoire et en politique et d'affirmer une identité républicaine qui n'existait guère $^{3}$. Au coté de cette prémisse, ce travail voulait aussi fournir du matériel aux écoles de déclamation et y former une esthétique du discours.

Dans l' Oratorum et rhetorum sententiae, divisiones, colores, nous rencontrons donc la double forme des déclamations telles qu'elles se présentent dans les écoles de rhétorique depuis le premier siècle avant notre ère ${ }^{4}$. Les Controverses sont composées de sententiae, divisiones et colores (les arguments des orateurs, le regroupement de ces arguments en un plan logique ainsi que les motifs indépendants de la loi allégués pour expliquer les actes de

Franz Steiner, 2003, p. 227-42. Sur la propagande officielle et des manifestations privées, voir notamment les œuvres de Zanker, P. The Power of Images in the Age of Augustus. Michigan: Michigan University, 1988 e Strothmann, M. Augustus - Vater de res publica. Sttugart: Franz Steiner, 2000.

2 Voir notamment l'introduction d'Henri Bornecque. Paris: Garnier frères, 1932, p. XVI-XVII et, plus récemment, Fairweather, J. Seneca the Elder. Cambridge: Univ. Press, 1981.

3 Liell, S. Die politischen Ambitionen des älteren Seneca. In: Czapala, B. (éd.). Vir bonus dicendi peritus: Festschrift für A. Weische, Wiesbaden: Rechert, 1997, p. 261-70.

4 Pour une étude de l'art oratoire à la fin de la République et les changements sous le Principat, voir notamment: Sinclair, P. Political declensions in Latin grammar and oratory 55 BCE - CE 39. Ramus. Monash University, n. 23, p. 92-109, 1994. Hellegouarc'h, J. Velleius Paterculus et Sénèque le Rhéteur. Remarques de langue et de style. In: Renard, M.; Laurens, P. (éds.), Hommages à Henry Bardon. Bruxelles: Ed. Latomus, 1985, p. 212-24. 
l'inculpé) $)^{5}$; Les Suasoires (suadere - conseiller, persuader) sont plus simples, faites pour persuader un personnage du discours et conseiller l'auditeur sur l'esthétique; elles ne comprennent pas de colores. L'organisation interne des chapitres suit donc généralement cet ordre: 1- le titre; 2- l'énoncé de la loi; 3- les sententiae; 4- la divisio; 5- les colores. Cette dernière partie n'existant pas pour les Suasoires.

Voyons les mentions que Sénèque fait d'Auguste. Cas d'une adoption d'un enfant né d'une courtisane, recueilli par son grand-père:

"Dans cette controverse, Latron dit quelque chose qui pouvait nuire, non pas à la cause, mais à lui-même. Il la déclamait devant César Auguste et $\mathbf{M}$. Agrippa, dont les fils, Lucius et Caïus, petit fils de l'empereur, devaient, selon toute apparence, être adoptés prochainement par celui-ci. M. Agrippa était de ceux qui ne sont pas nés nobles, mais le sont devenus. En parlant pour le jeune homme, et en développant le lieu commun de l'adoption, Latron dit: "Voici que celui-là, du plus bas rang, entre, par adoption, dans la noblesse" et bien d'autres choses du même goût. Mécène, en sifflant indiqua à Latron que l'empereur était pressé et qu'il eût à terminer sa déclamation. Certains voyaient là une méchanceté de Mécène, qui aurait essayé, en sifflant ainsi, non pas d'empêcher César d'entendre les paroles prononcées, mais de les lui faire remarquer. Mais, sous le divin Auguste, on jouit d'une telle liberté que, malgré la toute puissance que possédait alors M. Agrippa, il ne manqua pas de gens pour lui reprocher sa naissance. Il s'était appelé d'abord Vipsanius Agrippa, mais il avait supprimé le nom de Vipsanius, comme une preuve de l'humble rang de son père et se faisait appeler Marcus Agrippa." ${ }^{\circ}$

Au premier abord l'auditeur s'attend à ce que Latron soit blâmé par Auguste et par Agrippa. Mais bientôt Sénèque se fait plus précis et dit que celui qui pourrait se sentir gêné,

' La divisio généralement se compose de deux parties non nommées qui ont rapport, l'une au droit, l'autre à l'équité.

6 Toutes les traductions sont d'Henri Bornecque, op. cit., nota 2. Contrv. 2.4.12: In hac controversia Latro contrariam rem <non> controversiae dixit sed sibi. Declamabat illam Caesare Augusto audiente et M. Agrippa, cuius filios, nepotes suos, Caesar [Lucium et Gaium] adoptaturus diebus illis videbatur. Erat $M$. Agrippa inter eos qui non nati sunt nobiles sed facti. Cum diceret partem adulescentis Latro et tractaret adoptionis locum, dixit: 'iam iste ex imo per adoptionem nobilitati inseritur' <et> alia in hanc summam. Maecenas innuit Latroni festinare Caesarem; finiret iam declamationem. Quidam putabant hanc malignitatem Maecenatis esse; effecisse enim illum non ne audiret quae dicta erant Caesar, sed ut notaret. Tanta autem sub divo Augusto libertas fuit ut praepotenti tunc $M$. Agrippae non defuerint qui ignobilitatem exprobrarent Vipsanius Agrippa fuerat, <at> Vipsani nomen quasi argumentum paternae humilitatis sustulerat et $M$. Agrippa dicebatur. 
c'est Agrippa. Sénèque n'hésite pas à rappeler qu'Agrippa n'est pas noble de naissance mais l'est devenu; il n'hésite pas non plus à reproduire la phrase de Latron: "celui-là, du plus bas rang, entre par adoption dans la noblesse". Par analogie, Agrippa serait l'enfant de la courtisane adopté par Auguste. La suite, comme il arrive souvent chez Sénèque, est ambiguë: l'autre "méchant" de l'histoire est le fragile Mécène qui a sifflé à fin de réveiller Auguste pour qu'il intervienne en faveur d'Agrippa et de ses petits-fils.

Dans sa première mention d'Auguste, Sénèque nous montre donc que les citoyens pouvaient craindre l'empereur. Finalement, il ne s'agissait que d'un "mal entendu", car sous Auguste on aurait joui de liberté et on aurait pu reprocher à son général ses origines (et par conséquence celles des petits-fils d'Auguste). Ce passage nous montre précisément ceci: vers l'année 40 apr. J.-C. et avant, à l'époque où le texte se place, l'entourage d'Auguste n'était pas intouchable; les déclamateurs parlaient de l'origine d'Agrippa, ils regardaient Mécène avec méfiance; Sénèque n'hésite non plus à être direct: on pouvait "reprocher la naissance du puissant Agrippa" (M. Agrippae non defuerint qui ignobilitatem exprobrarent).

La deuxième mention du nom d'Auguste est apparemment fortuite: le divin Auguste fait l'éloge de L. Vinicius, reconnu par Sénèque comme très bon orateur": "C'est de lui que le divin Auguste a dit spirituellement: le talent de Vinicius, c'est de l'argent comptant." Il n'est pas impossible de voir une certaine ambiguité et de l'ironie dans la répétition de ces mots d'Auguste: le prince est montré avec son penchant pour l'argent; en effet, elegantia et numeratio sont des choses qui ne vont pas ensemble dans l'esprit romain.

Le prochain cas de mention directe d'Auguste se trouve dans une préface où Sénèque parle à ses enfants d'Asinius Pollion':

"Aussi quand, après la mort de C. César, décédé en Syrie, le divin Auguste, dans un billet écrit sur le ton affable et même amical qu'employait toujours ce prince si clément, se plaignit à lui [à Asinius Pollion qui venait de perdre son fils] de ce que, après un deuil si cruel pour lui et si récent, un homme qu'il aimait beaucoup avait dîné en nombreuse compagnie, Pollion répondit: "J'ai fait de même le jour où j'ai perdu mon fils Hérius". Comment exiger une douleur plus vive d'un ami que d'un père?"10

7 L. Vinicius pater, consul en 5 av.J.-C. (Tac. Ann.2. 11).

Contrv. 2.5.20: De hoc eleganter dixit divus Augustus: L. Vinicius ingenium in numerato habet.

Consul en 40 av. J.-C., auteur d'une Histoire qui traitait de 60 av. J-C. jusqu'à Philippes (42 av.J.-C.). Source de Plutarche.

10 Contrv. 4 préf.5: Itaque cum mortuo in Syria C. Caesare per codicillos questus esse divus Augustus, ut erat mos illi clementissimo viro, non civiliter tantum sed etiam familiariter, quod in tam magno et recenti luctu suo homo carissimus sibi pleno convivio cenasset, rescripsit Pollio: "eo die cenavi, quo Herium filium amisi." Quis exigerat majorem ab amico dolorem quam a patre ? Je laisse de coté la prochaine référence dans le même paragraphe: Telle était la rapidité de son élocution [d'Hatérius] qu'elle devenait un défaut. Aussi le divin Auguste a-t-il dit très justement de lui: "Notre bon Hatérius aurait besoin d'être enrayé," tellement il semblait ne pas 
Sénèque reconnaît la douleur d'Auguste et sa sensibilité envers Pollion. En effet, les deuils du prince étaient aussi des deuils publics, et l'image d'homme blessé est entrée dans la propagande d'Auguste. " Clementissimo uiro est peut-être une exagération quand on regarde les passages postérieurs de Sénèque sur les exactions des triumvirs.

La quatrième fois qu'Auguste est mentionné se trouve dans la préface de la dixième controverse. Sénèque digresse sur quelques orateurs et déclamateurs; il parle de Gavius Silon $^{12}$, avec lequel Auguste suivait de cours de rhétorique:

"Il déclama bien, ce Gavius Silon, à qui César Auguste, qui l'avait entendu souvent plaider dans la colonie de Tarragone, rendit pleine justice: il dit, en effet: "je n'ai jamais entendu père de famille plus éloquent." Il était homme à mettre en avant le père de famille et dissimuler l'orateur; il pensait qu'une partie de l'éloquence consiste à cacher l'éloquence."13

Auguste, comme Sénèque, reconnaît les qualités de Gavius Silon. Mais, Sénèque interprète les paroles du Princeps: le plus important pour Auguste est d'être un pater familias, condition avec laquelle le prince a joué à plusieurs reprises.

En digressant sur les "couleurs" d'une cause insolite ${ }^{14}$, Sénèque expose brièvement l'approche de plusieurs orateurs. Quand il parle d'un certain Craton ${ }^{15}$, il nous raconte une petite histoire de l'entourage de César:

"Il lui arrivait [à Craton] souvent d'entrer en discussion, devant César, avec Timagène, homme à la parole piquante et trop libre, sans doute parce que, durant beaucoup d'années, il ne l'avait pas été lui-même. [Timagène] devenu esclave cuisiner, de cuisinier porteur de litière, de ce poste il s'était levé jusqu'à l'amitié de César; mais il s'inquiétait si peu de ces deux conditions, celle où il se trouvait et celle où il s'était trouvé, que César, irrité

courir mais descendre une pente en courant." Tanta erat illi velocitas orationis ut vitium fieret. Itaque divus Augustus optime dixit: 'Haterius noster sufflaminandus est': adeo non currere sed decurrere videbatur (Contrv. 4 préf. 7).

"Sur le "temps du deuil" et la manipulation et conquête du temps civique, voir Fraschetti, A. Rome et il Principe. Roma-Bari: Laterza, 1990. Voir notamment RG. 14.

12 Déclamateur d'Espagne cité occasionnellement par Sénèque (Contrv. 10.1.7; 10.2.16; 10.3.14; 10.4.7; 10.5.1). L'épisode se passe à Tarraco. D'ailleurs inconnu.

${ }^{13}$ Contrv. 10. préf. 14: Bene declamavit Gavius Silo, cui Caesar Augustus, cum frequenter causas agentem in Tarraconensi colonia audisset, plenum testimonium reddidit; dixit enim: "nunquam audivi patremfamiliae disertiorem." Erat qui patremfamiliae praeferret, oratorem subduceret: partem esse eloquentiae putabat eloquentiam abscondere.

${ }^{14}$ Contrv. 10.5: Parrhasius, peintre athénien, mit un prisonnier à la torture et peignit un tableau de Prométhée. Le prisonnier trouva la mort. Parrhasius mit son tableau comme offrande dans le temple de Minerve. Il pourrait y avoir préjudice envers l'Etat.

is Orateur asiatique d'ailleurs inconnu. Cité aussi en Contrv.10.5.21. 
contre lui pour beaucoup de motifs, lui ayant interdit l'accès de sa maison, il brûla toute l'histoire qu'il avait écrite de ses actions comme pour lui interdire, à son tour, l'accès à son talent; c'était un homme éloquent et caustique; médisant, mais avec finesse."16

Auguste ne semble être lié à aucune considération ou remarque plus approfondie, mais c'est la lecture "rapide et efficace" de beaucoup d'historiens contemporains qui s'arrête sur ces apparences. Dans ce passage, tout comme dans le cas d'Agrippa (Contrv. 2.4.12), la préoccupation psycho-politique de Sénèque sur les origines des hommes est visible: il nous montre que les hommes proches du pouvoir n'ont pas eu de berceau. Il faudrait également remarquer que Sénèque n'omet pas de citer cette habitude, sur laquelle il reviendra, qu'ont les détenteurs du pouvoir de brûler des ouvrages. Mentionner ce fait, si l'on se rappelle de Cassius Severus ${ }^{17}$, était une critique directe contre Auguste. Quelques livres plus tard, l'auteur associera aussi les affranchis aux tyrans. Nous y reviendrons.

En lisant les Controverses nous pouvons constater que, dans les préfaces, Sénèque n'essaie pas de parler par d'autres; là, il ne s'agit pas de souvenirs, mais de sa propre voix adressée à ses auditeurs. C'est donc dans la dernière préface des Controverses ${ }^{18}$ qu'on pourrait attendre ses ultimes considérations personnelles sans la perturbation causée par d'autres voix dont nous ne connaîtrons jamais l'authenticité absolue. ${ }^{19}$

Sénèque parle de $\mathrm{T}$. Labienus $^{20}$, personnage qui a toute son admiration:

“C'est pour lui que l'on inventa un nouveau châtiment: les efforts de ses ennemis réussirent à faire condamner tous ses livres au feu; nouveauté

${ }_{16}$ Contrv. 10.5.22: Saepe solebat apud Caesarem cum Timagene confligere, homine acidae linguae et qui nimis liber erat: puto quia diu non fuerat. Ex captivo cocus, ex coco lecticarius, ex lecticario usque in amicitiam Caesaris enixus, usque eo utramque fortunam contempsit, et in qua erat et in qua fuerat, ut, cum illi multis de causis iratus Caesar interdixisset domo, combureret historias rerum ab illo gestarum, quasi et ipse illi ingenio suo interdiceret: disertus homo et dicax, a quo multa inprobe sed venuste dicta. Sur la recurrante antithèse de thèmes sociaux (les rapports entre affranchis, esclaves, patrons et autres) chez Sénèque, voir notamment Migliaro, E., Luoghi retorici e realtà sociale nell'opera di Seneca il Vecchio. Athenaeum. Università di Pavia, n. 67, p. 525-49, 1989.

17 Orateur exilé par Auguste en 12 ap. J.-C. par application de la Lex maiestas. Ses oeuvres ont été brûlées par ordre du Princeps (Tac. Ann.1.72.4.21). Cité aussi en Contrv. 3.préf.1. Voir notam. ment: Lassandro, D., La condanna di Cassio Severo. CISA. Vita e Pensiero, n. 22, p. 213-8, 1996.

${ }_{18}$ Les Suasoires n'ont pas de préface.

19 Sur la véracité du récit, les sources et la manipulation, voir notamment: Clausen, W., On Seneca the elder Suas. 5.2, AJPh. Johns Hopkins, n. 97, p. 1-2, 1976 et Locyer, C.W., The fiction of memory and the use of written sources. Convention and practice in Seneca the elder and other authors, Princeton: University, 1971.

${ }^{20}$ Titus Labienus. Orateur et déclamateur, ancien partisan de Pompée. Il a écrit aussi un pamphlet contre Bathylus, le favori de Mécènes. Ses ouvres interdites ont été restaurées par Gaius (Suet. Calig. 16). Cité aussi en Contrv. 10.2.19 et 10.3.5. 
inouïe que de soumettre ainsi des ouvrages au supplice! C'est vraiment un bonheur pour l'humanité que, du temps de Cicéron, l'on n'eût pas encore imaginé de tourner la cruauté contre les œuvres de l'esprit; que fût-il advenu, si les triumvirs avaient eu la fantaisie de proscrire le génie aussi de Cicéron? Les dieux immortels vengent lentement, mais sûrement, le genre humain; ils font retomber les châtiments extraordinaires sur la tête de ceux qui les ont imaginés, et, par une juste réciprocité de souffrances, ce que l'on avait inventé pour le supplice d'autrui, on l'expie souvent par le sien. Hommes pleins de folie, quelle démence vous agite? ... Les choses que la nature a mises à l'abri de toute souffrance, comme le génie et la mémoire, vous trouvez le moyen de leur faire subir aussi les maux qui torturent notre corps." 21

Dans ce riche passage, la mention de l'ancien partisan de Pompée est suivie d'une réflexion: les œuvres de Cicéron peuvent être brûlées! (Bono hercules publico ista in poenas ingeniorum versa crudelitas post Ciceronem inventa est; quid enim futurum fuit si triumviris libuisset et ingenium Ciceronis proscribere?). A cet holocauste, Sénèque répond en menaçant les triumvirs, ces hommes pleins de folie, de la vengeance divine ${ }^{22}$. "Ce que l'on avait invité pour le supplice d'autrui, on l'expie souvent par le sien", cela veut dire que les triumvirs seront suppliciés et qu'en plus, leurs ouvrages seront brûlés. Ces triumvirs qui veulent manipuler même la mémoire des hommes: sicut ingenium memoriamque nominis, invenite quemadmodum reducatis ad eadem corporis mala. Or, nous savons quel triumvir a laissé des ouvrages à ses contemporains et à la postérité. Parmi tous les textes que nous étudions, ce passage de Sénèque exprime peut-être le mieux l'opinion négative sur "I'Auguste triumvir", de même que l'imprécision entre la période triumvirale et celle d'une république restaurée. Par extension, l'auteur exprime ainsi son mécontentement envers Auguste et son régime. Nous y reviendrons. Il faudrait remercier Tibère de ne pas avoir brûlé ce passage.

${ }^{21}$ Contrv.10.préf.6: In hoc primum excogitata est nova poena; effectum est enim per inimicos ut omnes eius libri comburerentur: res nova et inusitata supplicium de studiis sumi. Bono hercules publico ista in poenas ingeniorum versa crudelitas post Ciceronem inventa est; quid enim futurum fuit si triumviris libuisset et ingenium Ciceronis proscribere? Sunt di inmortales lenti quidem sed certi vindices generis humani, et magna exempla in caput invenientium regerunt, ac iustissima patiendi vice quod quisque alieno excogitavit supplicio saepe expiat suo. Quae vos, dementissimi homines, tanta vecordia agitat? Parum videlicet in poenas notae crudelitatis est: conquirite in vosmet ipsos nova quibus pereatis, et si quid ab omni patientia rerum natura subduxit, sicut ingenium memoriam que nominis, invenite quemadmodum reducatis ad eadem corporis mala. H. Bornecque a traduit memoriamque par "gloire"; C'est le seul point où je juge nécessaire de signaler un désaccord avec sa traduction. Quelques lignes plus tard (Contrv. 10. Préf. 8), Sénèque dit que les livres ont été brûlés par ordre du Sénat (...illo tempore quo libri Labieni ex senatus consulto urebantur).

${ }^{2}$ La référence aux faits du passé se fait au temps présent. Cette atemporalité faisait naturellement partie de la théâtralité des exercices de déclamation. Voir à ce propos: Fairweather, J., The Elder Seneca and declamation, $A N R W 2$. De Gruyter, n. 32.1, p. 514-56, 1984. 


\section{Les Suasoires}

"Alexandre délibère s'il lancera ses navires sur l'Océan" La première Suasoire imagine un débat entre orateurs sur les avantages et les périls d'une telle aventure. Au premier abord, il s'agit là d'un de ces développements très éloignés de toute réalité historique. Mais Sénèque, homme rusé, en profite pour introduire dans ses commentaires (divisiones) sur les interventions des orateurs, ses critiques les plus remarquables contre deux attitudes qu'il combat: le pouvoir autoritaire et l'historiographie louangeuse. Dans le cas de ce passage, si nous nous rappelons de l'imitatio Alexandri chez César ${ }^{23}$ et du rapport Auguste-Alexandre, les propos de Sénèque deviendront plus clairs. Auguste est visé dans les digressions du discours. Voyons les premiers de mots de Sénèque à propos d'Alexandre:

"Cestius ${ }^{24}$ disait que ce genre de Suasoires devait être traité de façon différente suivant les endroits. Il ne fallait pas exprimer sa pensée dans une cité libre de la même façon que devant les rois, auxquels même des conseil utiles doivent être donnés sous une forme qui puisse leur plaire. Et parmi les rois mêmes, il y a des différences: ils détestent plus ou moins la vérité; or, sans conteste, Alexandre est de ceux que l'histoire nous a présenté comme plein de suffisance et gonflé d'un orgueil outrecuidant pour un mortel. Dans tous les cas, même en laissant de côté les autres preuves, la Suasoire met en lumière son arrogance: son monde ne lui suffit plus. Aussi Cestius disait-il qu'il fallait dans tout les discours marquer le plus grand respect pour le roi, afin d'éviter le sort de son censeur, cousin du précepteur Aristote, qu'il tua pour des railleries plus hardies qu'il n'aurait convenu; comme Alexandre, qui voulait se faire passer par dieu, avait été blessé, le philosophe en voyant son sang, dit qu'il s'étonnait que ce ne fût pas "ce sang limpide qui coule dans les veines des dieux bienheureux". Le roi se vengea de cette plaisanterie par un coup de lance." ${ }^{25}$

${ }^{23}$ César est presque indemne des critiques de Sénèque et est toujours mentionné laconiquement (Contrv. 7.3.9; 10 préf.16; 10.3.1. Suas. 6.12; 7.1.25). Malgré l'intérêt que cela peut susciter, une étude approfondie de l'image de César et des comparaissons avec Auguste, cela sera l'objet d'une autre recherche. Sur l'imitatio Alexandri chez César et Auguste voit notamment Martin, P.-M., L'autre héritier de César. In: Marc Antoine, son ideologie et sa descendance, Actes du Colloque organisé a Lyon le jeudi 28 juin 1990. Paris: De Boccard, 1993, p. 37-54.

Lucius Cestius Pius, né à Smyrne: Il possédait une des écoles les plus populaires à Rome. Souvent critiqué par Sénèque pur son abus des traits (Contrv. 3 préf. 15; 7.1 .27; 7.8; 8.6.12. Suas. 1.5.6; 7.12).

Suas. 1.5.1: Aiebat Cestius hoc genus suasoriarum <alibi> aliter declamandum esse [quam suadendum]. Non eodem modo in libera civitate dicendam sententiam quo apud reges, quibus etiam quae prosunt ita tamen ut delectent suadenda sunt. Et inter reges ipsos esse discrimen: quosdam minus, alios magis veritatem pati; Alexandrum ex iis esse quos superbissimos et supra mortalis animi modum inflatos accepimus. Denique, ut alia dimittantur argumenta, ipsa suasoria insolentiam eius coarguit; orbis illum suus non capit. Itaque nihil dicendum aiebat nisi cum summa veneratione regis, ne accideret idem quod praeceptori eius, amitino Aristotelis, accidit, quem occidit propter intempestive liberos sales; nam cum se deum vellet videri et vulneratus 
Alexandre, un de ceux qu'enfle "un orgueil outrecuidant pour un mortel" est un modèle d'Auguste. Celui à qui Auguste a rendu hommage en Egypte est un arrogant: "même en laissant de côté les autres preuves, la Suasoire met en lumière son arrogance: son monde ne lui suffit plus." Dans la suite de ces lignes le roi "divin" punit de mort un philosophe; il s'agit d'un roi devant lequel il faut peser les mots. Si, par ailleurs, nous nous rappelons de Cicéron et du banquet des divinités où Auguste s'est travesti en Apollon ${ }^{26}$, considérons cette analogie pour l'instant comme trop forcée et non valide. Voyons la suite du texte:

"Cette anecdote est rappelée avec finesse dans une lettre de C. Cassius ${ }^{27}$ à M. Cicéron: il y plaisante longuement sur la sottise du jeune Pompée, qui rassembla une armée en Espagne et fut vaincu à la bataille de Munda; puis il ajoute: "Nous nous moquons de lui, mais je crains qu'il ne nous retourne nos moqueries à la pointe de son épée." Chez tous les rois il faut redouter cette façon de plaisanter." 28

Auparavant Sénèque avait présenté un roi divin qui exécute un philosophe. Ici, en parlant de Cicéron, il qualifie Pompée de rex; Il montre par là que la royauté n'est pas seulement orientale (In omnibus regibus haec urbanitas extimescenda est). Dans la suite du texte l'auteur revient à la question de l'attitude à avoir devant Alexandre:

"Aussi disait-il [Cestius] que devant Alexandre il importait d'exprimer sa
pensée en termes dont l'adulation profonde chatouillerait agréablement son
esprit: toutefois il convenait de garder une certaine mesure pour se donner
l'air de le respecter et non de le flatter..."29

Il est difficile de ne pas penser à Auguste quand on se souvient des citations antérieures. ${ }^{30}$ Dans la suite de cette "anecdote" (fabellarum) comme il l'appellera plus tard, Sénèque revient à la Rome des triumvirs; l'auteur nous narre l'épisode d'Antoine "dieu Bacchus" à Athènes et nous parle d'un personnage, Dellius:

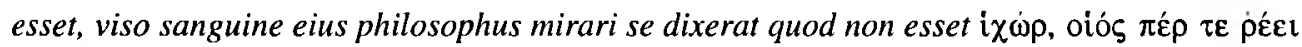
$\mu \alpha \kappa \alpha \rho \varepsilon \sigma \sigma \iota \mathrm{\theta} \theta \hat{i} \sigma \mathrm{\imath}$. Ille se ab hac urbanitate lancea vindicavit.

${ }^{26}$ Suet. Div. Aug.70.

${ }^{27}$ Gaius Longuinus Cassius, tyrannicide (Suas. 6.11.17; 7.5).

${ }^{28}$ Suas 1.5.20: Eleganter in C. Cassi epistula quadam ad M. Ciceronem missa positum: multum iocatur de stultitia Cn. Pompei adulescentis, qui in Hispania contraxit exercitum et ad Mundam acie victus est; deinde ait: "nos quidem illum deridemus, sed timeo ne ille nos gladio

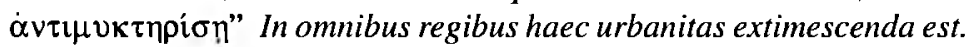

29 Suas. 1.6: Aiebat itaque apud Alexandrum esse <sic> dicendam sententiam ut multa adulatione animus eius permulceretur, servandum tamen aliquem modum, ne non veneratio <videretur sed adulatio $>$...

${ }^{30}$ Notamment dans la Controverse 2.4.12, voir note 6. Aussi la Contrv. 6.8 Paragraphe final: "En dehors. Varius Geminus dit devant César: "César, ceux qui osent parler devant toi ignorent ta grandeur, ceux qui n'osent pas, ta bonté”. Dans ce cas c'est Jules César. 
"Cependant il y eu un très joli mot de Dellius, que Messala Corvinus ${ }^{31}$ appelle l'acrobate des guerres civiles, parce que, sur le point de passer de Dolabella ${ }^{32}$ à Cassius, celui-ci spécifia qu'il aurait la vie sauve s'il tuait Dolabella; puis de Cassius il passa à Antoine, et en dernier lieu, il abandonna Antoine pour César." ${ }^{33}$

Dans le thème de la royauté nous avons une nouvelle confusion temporelle où nous retrouvons Alexandre, Pompée, Antoine et enfin Auguste; on le voit accueillir des opportunistes dans son rang. Avec ces références croisées entre Alexandre et l'histoire récente de Rome, l'auteur trace un parallélisme historique entre Alexandre et le Principat d'Auguste; quand il parle des cités libres, d'Alexandre, des rois (au pluriel), de Cicéron et de Marc Antoine, il laisse entrevoir que Rome n'est pas une cité libre et qu'Auguste est un roi. Et il y profite pour s'attaquer aux gens du parti au pouvoir.

Dans les deux dernières Suasoires, les orateurs et déclamateurs parleront encore de Cicéron $^{34}$, en citant nommément Antoine et Lépide, mais jamais Auguste. Quand ils doivent se référer aux actions du jeune Octave, ils diront "les triumvirs". Ce sont ces contours et détours que les modernes ont appelés "l'ironie" de Sénèque. Il faudrait remarquer que, s'il est certain qu'Auguste n'est jamais nommément critiqué, il n'en va pas de même pour le "dieu César": "L'esclavage, il (Cicéron) ne le refusera pas; il a déjà le cou tout usé par la chaîne: Pompée d'abord, César ensuite l'ont soumis; voyez en lui un vétéran de l'esclavage."'35

L'habitude qu'a l'historien de contextualiser les auteurs qu'il étudie ne doit pas jouer un rôle de prédéfinition et prévision de ce qui est écrit; trop insister sur les déterminants géographiques, sociaux ou politiques, ce serait simplifier une réalité complexe qui restera toujours inatteignable dans sa totalité; ce serait ignorer l'individu en tant qu'agent historique et appauvrir l'histoire; il s'agirait d'ailleurs de vains exercices de clairvoyance. Laissons Sénèque se contextualiser lui-même:

"Ensuite vous pourrez peser dans quelle mesure le talent diminue chaque jour, et je ne sais pas quelle défaveur de la nature, l'éloquence a rétrogradé:

31 Marcus Valerius Messala Corvinus a proposé le titre de pater patriae à Auguste (Suet. Div. Iul. 53); Loué par Sénèque (Contrv. 2.4.8; 3. préf. 14; Suas. 1.8; 2.17; 3.6; 6.27). Sur ce personnage voir notamment: Valvo, A. Dans l'ombre des plus grands: I poètes et prosateurs mal connus de l'époque augustéenne. ANRW 2. De Gruyter, n. 30.3, p. 1663-80, 1982.

Suas. 1.7: Bellissimam tamen rem Dellius dixit, quem Messala Corvinus desultorem bellorum civilium vocat quia ab Dolabella ad Cassium transiturus salutem sibi pactus est si Dolabellam occidisset, a Cassio deinde transit ad Antonium, novissime ab Antonio trans fugit ad Caesarem. Sur les trois versions de la mort de Cicéron, la façon dont elles sont narrées par leur développement dans l'historiographie ancienne, voir notamment: Roller, M.B. Color-Blindness: Cicero's death, declamation and the production of History Cph. University of Chicago, n. 92, p. 109-30, 1997. 
tout ce que l'éloquence romaine pouvait mettre à côté ou au-dessus de l'orgueilleuse Grèce, a fleuri vers l'époque de Cicéron; alors parurent les talent auxquels l'art que nous étudions doit tout son éclat. Puis chaque jour nous a emportés vers la décadence, soit par suite du luxe de notre époque (car rien n'est si funeste à l'éloquence que le luxe); soit que les récompenses attachées à cet art si beau ayant disparu, toute l'émulation se soit portée vers des occupations honteuses, que mettent en vogue des honneurs et des profits; soit par une fatalité, dont la loi jalouse et éternelle veut que tout ce qui est parvenu au faîte retombe jusqu'en bas, et d'une chute plus rapide que la montée." 36

Louer Auguste, depuis Virgile, revient à louer son époque. L'époque de Sénèque, d'Auguste et de Tibère est, selon l'auteur, l'époque de la décadence, du luxe, des occupations honteuses, des profits. La propagande d'Auguste ne montre ici aucun effet. Sénèque at-il des contemporains valeureux ? "Lequel de nos contemporains est, dirai-je l'esprit assez meublé ou assez travailleur? Non, lequel est assez homme?" (Quis aequalium vestrorum quid dicam satis ingeniosus, satis studiosus, immo quis satis vir est? Contrv.1.Préf.9). Le régime d'Auguste et de Tibère ne pense pas à préserver la mémoire des grands déclamateurs: "je rendrai ainsi, je crois, un grand service à ces orateurs mêmes; l'oubli les menace, si l'on ne transmet à la postérité quelque chose qui prolonge leur mémoire. En effet, il n'existe pour ainsi dire pas de recueil des œuvres des plus grands déclamateurs, ou ceux qui existent sont apocryphes, ce qui est pire". ${ }^{37}$ C'est pour cette raison que l'auteur travaille sur un nouveau genre: "Mais les déclamations de Cicéron ne ressemblaient pas à ce que nous appelons controverses, ni même aux exercices oratoires usités avant lui qu'on nommait thèses. Car le genre précis, qui sert à nos exercices, est si nouveau que le non même est nouveau". ${ }^{38}$ Dans le même sens, la récurrante loyauté de l'auteur envers les ennemis des Césars, exprime sa critique et son opposition politique. ${ }^{39}$

${ }^{36}$ Contrv. 1.Préf. 6: Non est unus, quamvis praecipuus sit, imitandus, quia numquam par fit imitator auctori. Haec rei natura est: semper citra veritatem est similitudo. Deinde ut possitis aestimare in quantum cotidie ingenia decrescant et nescio qua iniquitate naturae eloquentia se retro tulerit: quidquid Romana facundia habet quod insolenti Graeciae aut opponat aut praeferat circa Ciceronem effloruit; omnia ingenia quae lucem studiis nostris attulerunt tunc nata sunt. In deterlus deinde cotidie data res est, sive luxu temporum nihil enim tam mortiferum ingeniis quam luxuria est sive, cum pretium pulcherrimae rei cecidisset, translatum est omne certamen ad turpia multo honore quaestuque vigentia, sive fato quodam, cuius maligna perpetuaque in rebus omnibus lex est ut ad summum perducta rursus ad infimum, velocius quidem quam ascenderant, relabantur.

37 Contrv. 1 préf. 11: Ipsis quoque multum praestaturus videor, quibus oblivio imminet, nisi aliquid, quo memoria eorum producatur, posteris traditur: fere enim aut nulli commentarii maximorum declamatorum exstant aut, quod pejus est, falsi.

38 Contrv.1 préf.12: Declamabat autem Cicero non quales nunc controversias dicimus, ne tales quidem, quales ante Ciceronem dicebantur, quas thesis vocabant. Hoc enim genus maxime, quo nos exercemur, adeo novum est, ut nomen quoque ejus novum sit.

39 Iunius Gallio. Chassé du Sénat par Tibère (Tac. Ann. 6,3; Sen. Contrv. 7 préf.15; 10, préf. 8 et 13); Votienus Montanus, exilé par Tibère en 4 ap. J.-C., (Contrv. 9.14.11-16; Tac. Ann. 4.42); Cassius Severus, voir note 17; Titus Labienus, voir note 20. 
Le monde de Sénèque n'est pas aussi simple que le dépeignent les Res Gestae d'Auguste, où nous lisons l'histoire du "Bien triomphant" et de la "république restaurée". Quand Sénèque juge l'assassin du tyran, il dit, par Pompeius Silon, que cet assassin doit être poursuivi s'il haît la liberté publique (Contrv. 1.7.13); tuer le tyran n'est pas en soi un acte glorieux; tel acte doit être inspiré par l'amour de la patrie (Contrv. 4.7.2 excerpta). Dans un autre cas, l'homme qui répudie sa femme stérile, peut être même plus cruel que le tyran (Contrv. 2.5.9). ${ }^{40}$ Dans ce monde que les orateurs et les déclamateurs de Sénèque veulent montrer beaucoup plus complexe que ne la dépeint la propagande des détenteurs du pouvoir, même les pirates peuvent être miséricordieux (Contrv. 1.7.3).41

\section{Conclusion}

A la question "a-t-on le droit d'attaquer [devant les tribunaux] des actions faites durant les guerres civiles ?" (Contrv.7.2.9) posée par Sénèque, et non par un de ses déclamateurs, il ne donne pas de réponse. Son silence réfléchi les limites qui s’imposaient.

Hommes de leur temps, même si les déclamateurs et orateurs cherchaient des sujets abstraits pour leurs exercices de culture et d'identité, leur raisonnement de base était leur réalité politique. C'est cette réalité qui explique la récurrence dans les textes de cas comme ceux de la tyrannie, avec des problématiques qui ne sont pas simples (la question de savoir s'il existe des crimes plus graves que ceux du tyran revient plusieurs fois). De même, quand un déclamateur parle de l'affranchissement des esclaves, il expose ce fait comme un acte politique typique du tyran: "Venez sur ces bancs, esclaves affranchis, mes nouveaux parents que j'ai achetés" (in illa subsellia transite servi, transite liberti, empta cognatio. Contrv. 7.6.24). Or, nous savons quels chefs politiques de l'époque de Sénèque affranchissaient des esclaves.

Par rapport aux grands thèmes de la propagande d'Auguste, époque durant laquelle l'auteur a vécu, nous ne la trouvons guère chez Sénèque: pas un mot sur le Saeculum novum, ni sur la res publica restituta. Tibère, le dernier dauphin attitré, est cité très brièvement quatre fois, sans jamais recevoir un éloge (Contrv. 7.1.27; 7.5.11; 9.4.19; Suas. 3.6). De même, très peu de mots sur d'autres membres de la famille impériale, aucun deuil pour les morts; de façon remarquable, c'est justement à cette époque où le pouvoir des femmes au

40 Sur la problématique du tyran voir notamment: Tabacco, R., Il tiranno nelle declamazioni di scuola in lingua latina. MAT. Accademia delle scienze di Torino, n. 9, p. 1-141, 1985. Il faudrait signaler qu'Auguste est plus associé aux rois et moins aux tyrans. Sur la relativité de la virtus: Lentano, M., Del bon uso delia virtù: due note alla controversiae 1,8 di Seneca il Vecchio. Aufidus. Atlantica, n. 24, p. 25-33, 1994.

${ }^{41}$ Les personnages de pirates et de voyageurs figurent au premier plan dans les Controverses (1.2; $1.6 ; 1.7 ; 7.1$ ). Quoi qu'il s'agisse de thèmes d'école, dont beaucoup sont empruntés à la Grèce, ces déclamations ne sont pas totalement éloignées de la réalité: elles nous permettent de mesurer les risques auxquels s'exposait tout voyageur à l'époque d'Auguste. Voir notamment Chambert, R. Pirates et voyageurs dans les Controverses de Sénèque le Père. REL, Les Belles Lettres, n. 77, p. 149-69, 1999. 
sein de la famille impériale prend de l'essor, que Sénèque ose dire: "Malheur à celui qui offense cette courtisane! Malheur à la mère de famille, dont la beauté fait ombrage à cette courtisane! Quoi qu'elle demande, le préteur ne dira jamais non." ( $O$ miserum, si quis meretricem offendit! o miseram matrem familiae, si quoius formae meretrix invidet! Nihil petenti praetor negaturus est. Contrv. 9.2.1). Néanmoins, au plan des apparences, la propagande d'Auguste a partiellement réussi à provoquer chez Sénèque une séparation entre la période totalement tyrannique des triumvirs et une autre "sous tutelle". Cette seconde période, dont l'auteur ne précise jamais la frontière, est le temps d'Auguste clementissimo viro, mais, remarquons, elle est aussi le luxus temporum, la période de la malarum rerum industria, des obscena studia et du comportement nisi in libidine. (Contrv. 1. pr. 10.2).

Avec la relativité de ses causes, Sénèque invite ses lecteurs à réfléchir, il leur montre qu'il existe une réalité complexe derrière la façade du discours officiel. La "fin des guerres civiles", la "lutte contre Marc Antoine", ces mots, ne justifient pas en eux-mêmes les actes de certains. Par le biais de références à d'autres époques et d'autres personnages, il peut affirmer des sentences telle que "les actes imposés par la violence et la crainte seront nuls" (Per vim metumque gesta irrita sint. Contrv. 4.8 excerpta); cet énoncé auquel Sénèque veut donner la valeur de loi, a été cité précisément dans un épisode de guerre civile et de proscription. "Il sera permis à un candidat de parler contre son compétiteur" (competitori liceat in competitorem dicere. Contrv. 5.8 excerpta); cet autre énoncé se réfère au cas d'un tyran qui brigue une magistrature après avoir obtenu l'amnistie du passé; l'orateur dit au tyran: "Au nom de la liberté publique, je t'en conjure, ne me fais pas condamner à mort: on t'accusera d'avoir tué un citoyen" (Per communem deprecor libertatem, ne moriar: objicietur tibi, quod occideris civem. (Contrv. 5.8 excerpta); ce sont les paroles que, par la voix de Sénèque, tous les orateurs et déclamateurs adressent à Auguste et Tibère.

$$
* * *
$$

\section{Fontes}

AUGUSTO. Res gestae divi Augusti. Tradução de Jean Gagé. Paris: Belles Lettres, 1977. SENECA O VELHO. Controversiae et Suasoriae. Tradução de Henri Bornecque. Paris: Garnier Frères, 1932.

\section{Referências Bibliográficas}

CHAMBERT, R. Pirates et voyageurs dans les Controverses de Sénèque le Père. $R E L$, Les Belles Lettres, n. 77, p. 149-69, 1999.

CLAUSEN, W. On Seneca the elder Suas. 5.2. AJPh. Johns Hopkins, n. 97, p. 1-2, 1976.

FAIRWEATHER, J. The Elder Seneca and declamation. ANRW 2. De Gruyter, n. 32.1, p. 514-56, 1984. 
. Seneca the Elder. Cambridge: Univ. Press, 1981.

FRASCHETTI, A. Rome et il Principe. Roma-Bari: Laterza, 1990.

HELLEGOUARC'H, J. Velleius Paterculus et Sénèque le Rhéteur. Remarques de langue et de style. In: RENARD, M.; LAURENS, P. (éds.) Hommages à Henry Bardon. Bruxelles: Ed. Latomus, 1985, p. 212-4.

LASSANDRO, D. La condanna di Cassio Severo. CISA. Vita e Pensiero, n. 22, p. 213-8, 1996. LENTANO, M., Del bon uso della virtù: due note alla controversiae 1,8 di Seneca il Vecchio. Aufidus. Atlantica, n. 24, p. 25-33, 1994.

LIELL, S. Die politischen Ambitionen des älteren Seneca. In: CZAPALA, B. (éd.). Vir bonus dicendi peritus: Festschrift für A. Weische, Wiesbaden: Rechert, 1997, p. 261-70.

LOCYER, C.W., The fiction of memory and the use of written sources. Convention and practice in Seneca the elder and other authors, Princeton: University, 1971.

MALITZ, J. Autobiographie und Biographie römischer Kaiser im 1. Jh. n. Chr. In: WEBER, G. (Org). Propaganda - Selbstdarstellung - Repräsentation in römischen Kaiserreich des 1. Jhs. n. Chr. Stuttgart: Franz Steiner, 2003, p. 227-42.

MARTIN, P.-M. L'autre héritier de César. In: Marc Antoine, son idéologie et sa descendance, Actes du Colloque organisé à Lyon le jeudi 28 juin 1990. Paris: De Boccard, 1993, p. 37-54.

MIGLIARO, E. Luoghi retorici e realtà sociale nell'opera di Seneca il Vecchio. Athenaeum. Università di Pavia, n. 67, p. 525-49, 1989.

ROLLER, M.B. Color-Blindness: Cicero's death, declamation and the production of History. Cph. University of Chicago, n. 92, p. 109-30, 1997.

SINCLAIR, P. Political declensions in Latin grammar and oratory 55 BCE - CE 39. Ramus. Monash University, n. 23, p. 92-109, 1994.

STROTHMANN, M. Augustus - Vater de res publica. Sttugart: Franz Steiner, 2000.

TABACCO, R., Il tiranno nelle declamazioni di scuola in lingua latina. MAT. Accademia delle scienze di Torino, n. 9, p. 1-141, 1985.

VALVO, A. Dans l'ombre des plus grands: I poètes et prosateurs mal connus de l'époque augustéenne. ANRW 2. De Gruyter, n. 30.3, p. 1663-80, 1982.

ZANKER, P. The Power of Images in the Age of Augustus. Michigan: Michigan University, 1988.

TORRI, Marcos. La réception de la propagande d'Auguste chez Sénèque le Rhéteur.Classica, São Paulo, 15/16, p. 117-130, 2002/2003.

Résumé: Les ambiguïtés et les parallélismes historiques de Sénèque l'Ainé dans ses mentions à Auguste, démontrent que la mémoire du Princeps n'entre pas dans le cadre des grands thèmes de la propagande impériale. Par ces moyens, l'auteur exprime une sorte d'opposition politique.

Mots-clés: Rome; Principat; Auguste; Sénèque l'Aîné; mémoire; propagande; opposition politique. 